\title{
Evidence for altered hepatic gluconeogenesis in patients with cirrhosis using in vivo 31-phosphorus magnetic resonance spectroscopy
}

\author{
K K Changani, R Jalan, I J Cox, M Ala-Korpela, K Bhakoo, S D Taylor-Robinson, J D Bell
}

Magnetic Resonance Unit, MRC Clinical Sciences Centre, Imperial College School of Medicine, Hammersmith Hospital, London, UK K K Changani R Jalan† M Ala-Korpela J D Bell

Department of Imaging, Division of Investigative Sciences, Imperial College School of Medicine, Hammersmith Hospital, London, UK I J Cox

Division of Medicine (Medicine A), Imperial College School of

Medicine,

Hammersmith Hospital, London, UK S D Taylor-Robinson

MRC Biochemical and Clinical Magnetic Resonance Unit, Department of Biochemistry, University of Oxford, 30 South Parks Road, Oxford OX1 3QU, UK K Bhakoo

†Present address: Institute of Hepatology, University College London, 69-75 Chenies Mews, London WC1E 6HX, UK

Correspondence to: Dr S D Taylor-Robinson, Magnetic Resonance Unit, MRC Clinical Sciences Centre, Imperial College School of Medicine, Hammersmith Hospital, Du Cane Road, London W12 ONN, UK.

s.taylor-robinson@ic.ac.uk

Accepted for publication 26 February 2001

\begin{abstract}
Background and aims-Alterations in gluconeogenesis in the diseased liver can be assessed non-invasively using magnetic resonance spectroscopy by measuring changes in phosphomonoester resonance which contains information regarding several metabolites, including the phosphorylated intermediates of the gluconeogenic pathway.

Methods - ${ }^{31} \mathbf{P}$ magnetic resonance spectroscopy was used to determine changes in phosphomonoesters following bolus infusions of $2.8 \mathrm{mmol} / \mathrm{kg} \mathrm{L}$-alanine in five patients with functionally compensated cirrhosis and in five patients with functionally decompensated cirrhosis.

Results-Compared with six healthy volunteers, baseline phosphomonoester values were elevated by $35 \%(p<0.05)$ in the compensated cirrhosis group and by $57 \%(p<0.01)$ in the decompensated cirrhosis group. Following alanine infusion, phosphomonoesters in healthy volunteers increased by $46 \%$ from baseline values $(p<0.01)$, in patients with compensated cirrhosis by $27 \%(p<0.02)$ but those with decompensated cirrhosis showed no increase from baseline. There was a reduction in the percentage of inorganic phosphate signal in all subjects.

Conclusions-By analysing changes in phosphomonoester and inorganic phosphate resonances it is possible to discern clear metabolic differences between healthy volunteers and patients with cirrhosis of varying severity using magnetic resonance spectroscopy. Those patients with functionally decompensated cirrhosis have higher percentage baseline phosphomonoester values but the absence of phosphomonoester elevation following L-alanine infusion suggests that they are unable to mount a significant metabolic response with a progluconeogenic stimulus.

(Gut 2001;49:557-564)
\end{abstract}

Keywords: gluconeogenesis; cirrhosis; alanine; ammonia; glucagon

Gluconeogenesis is a pathway of key importance to humans, being essential for maintaining the supply of glucose to the brain and muscle. Derangements of carbohydrate metabolism in cirrhosis are manifest as progressive glucose intolerance but in decompensated patients there may be acute post absorptive hypoglycaemia, primarily due to a reduction in hepatic glycogen capacity. ${ }^{12}$ The mechanisms of glucose intolerance in chronic liver disease are not completely characterised but could be due to one or more factors such as altered glycolytic enzyme activity, changes in specific glucose transporter expression, reduced insulin production, or impairment of membrane receptors for insulin. Resting energy expenditure is increased ${ }^{3}$ in patients with cirrhosis but the respiratory quotient is reduced, indicating the use of fat stores as an energy source. ${ }^{4}$ This requirement for such an alternative energy source probably arises because there are reduced hepatic glycogen stores. ${ }^{5}{ }^{6}$ The need to maintain normal hepatic glucose output results in the observed increase in gluconeogenesis. ${ }^{6-8}$ However, the response of the cirrhotic liver to an amino acid challenge is not fully determined in humans.

In vivo phosphorus-31 magnetic resonance spectroscopy ( $\left.{ }^{31} \mathrm{P} \mathrm{MRS}\right)$ is a non-invasive technique which can be used to measure certain phosphorylated compounds, including the phosphorylated intermediates of the gluconeogenic pathway. At clinical magnetic field strengths, some of the ${ }^{31} \mathrm{P} M R$ resonances observed are multicomponent, the in vivo ${ }^{31} \mathrm{P}$ MR spectrum of the human liver containing phosphomonoester (PME), phosphodiester $(\mathrm{PDE})$, inorganic phosphate $\left(\mathrm{P}_{\mathrm{i}}\right)$, and three nucleotide triphosphate (NTP) resonances. ${ }^{9}$ The PME resonance includes contributions from precursors of phospholipid membrane synthesis (phosphocholine (PC) and phosphoethanolamine (PE)) as well as contributions from adenosine monophosphate (AMP) and gluconeogenic intermediates such as glucose-6-phosphate (G6P) and 3-phosphoglycerate (3PG). ${ }^{10}$

L-alanine has been shown in animal studies to increase gluconeogenic rates following its conversion to pyruvate. ${ }^{11}$ In our previous work we demonstrated that following an intravenous bolus injection of L-alanine there were significant changes in the in vivo hepatic ${ }^{31} \mathrm{P} M R$ spectra of healthy volunteers. ${ }^{9}$ Increases in PME resonance were observed which were

Abbreviations used in this paper: AMP, adenosine monophosphate; AUC, area under the curve; BLAST, baseline artefact subtraction technique; G6P,

glucose-6-phosphate; MRS, magnetic resonance spectroscopy; NTP, nucleotide triphosphate; PBC, primary biliary cirrhosis; PC, phosphocholine; PE, phosphoethanolamine; $\mathrm{P}_{\mathrm{i}}$, inorganic phosphate; PDE, phosphodiesters; PME, phosphomonoesters; 2D-CSI, two dimensional chemical shift imaging; 3PG,

3-phosphoglycerate. 
Table 1 Liver function tests, prothrombin time, and Pugh's score of study patients on the day of in vivo hepatic ${ }^{31} P M R S$ examination

\begin{tabular}{|c|c|c|c|c|c|c|}
\hline Patient group & $\begin{array}{l}\text { Plasma alanine } \\
\text { transaminase }(u / l) \\
(5-40)^{3}\end{array}$ & $\begin{array}{l}\text { Plasma alkaline } \\
\text { phosphatase (u/l) } \\
(35-130)^{3}\end{array}$ & $\begin{array}{l}\text { Plasma bilirubin } \\
(\text { mmol/l) } \\
(5-17)^{3}\end{array}$ & $\begin{array}{l}\text { Plasma albumin } \\
(\mathrm{g} / \mathrm{l}) \\
(35-50)^{3}\end{array}$ & $\begin{array}{l}\text { Prothrombin time } \\
\text { (s) } \\
(12-14)^{3}\end{array}$ & $\begin{array}{l}\text { Pugh score } \\
(5-15)\end{array}$ \\
\hline $\begin{array}{l}\text { Compensated } \\
\text { cirrhosis }(n=5)^{1}\end{array}$ & $55(32-76)$ & $233(82-319)$ & $24(7-71)$ & $41(39-43)$ & $13.5(12-15)$ & $6(5-7)$ \\
\hline $\begin{array}{l}\text { Decompensated } \\
\quad \text { cirrhosis }(n=5)^{2}\end{array}$ & $72(30-155)$ & $205(110-515)$ & $158(27-367)$ & $33(28-40)$ & $18.5(16-19)$ & $10(9-11)$ \\
\hline
\end{tabular}

Median (range) values.

${ }^{1}$ Compensated cirrhosis $=$ Pugh's score $\leqslant 7 ;^{2}$ decompensated cirrhosis=Pugh's score $\geqslant 8$.

${ }^{3}$ Normal range.

attributed to altered gluconeogenesis. Confirmatory in vivo animal studies revealed that these PME changes in the healthy liver following a progluconeogenic stimulus with an intravenous L-alanine infusion were caused by increased gluconeogenic precursors, such as 3PG and G6P. ${ }^{12}$ Simultaneous in vivo hepatic ${ }^{31} \mathrm{P}$ MRS and glucose turnover measurements using labelled glucose and alanine in patients with cancer cachexia has demonstrated that the elevated hepatic PME levels observed following an alanine challenge are a result of increased glucose flux and gluconeogenesis from alanine. ${ }^{13}$

In this study we used in vivo hepatic ${ }^{31} \mathrm{P}$ MRS to delineate spectral changes which can be used to provide insight into altered hepatic gluconeogenesis. In light of previous ${ }^{31} \mathrm{P}$ MRS studies of the liver following a progluconeogenic stimulus with L-alanine, ${ }^{912}{ }^{13}$ we wished to test the hypothesis that gluconeogenesis is significantly impaired following intravenous L-alanine challenge in patients with functionally decompensated chronic liver disease. We aimed to establish whether ${ }^{31} \mathrm{P}$ MR spectral patterns could be distinguished between patients with functionally compensated and decompensated cirrhosis, reflecting differences in gluconeogenic rates in the two groups of patients. Results were compared with data from matched normal healthy volunteers.

\section{Materials and methods}

The study was carried out in accordance with the Declaration of Helsinki (1989) of the World Medical Association and prior ethics approval was obtained from the ethics committee of Hammersmith Hospital, London, UK (REC 93/4239). All subjects provided written informed consent. Individuals with claustrophobia, cardiac pacemakers, or magnetic implants were excluded from the study. Each study was undertaken after a 12 hour overnight fast.

SUBJECTS

Six healthy volunteers (mean age 48 (range 32-63) years) with no clinical or biochemical evidence of liver disease were studied. None was taking regular medication and all consumed less than $20 \mathrm{~g}$ of alcohol per day. All were abstinent from alcohol for at least three days prior to the study.

All patients had clinically stable biopsy proved cirrhosis. Three patients (30\%) had primary biliary cirrhosis (PBC) and seven $(70 \%)$ alcohol related cirrhosis (abstinent for at least six months). Liver function was assessed using the standard Pugh score ${ }^{14}$ which grades liver function in patients with cirrhosis, ranging from a score of 5 (best or normal function) to 15 (worst function). Data were acquired on the day of the MRS study (table 1). Patients were divided into two groups (table 1): those with a Pugh score $\leqslant 7$ (mean 6) were classified as having functionally compensated cirrhosis (four males, one female). Three patients had alcohol related cirrhosis and two had PBC. They did not have hepatic encephalopathy or ascites and had more modest impairment of liver function. Their mean plasma urea level was 5.6 (range 4.3-6.7) $\mathrm{mmol} / \mathrm{l}$. Those with a Pugh score $\geqslant 8$ (mean 10) were classified as having functionally decompensated cirrhosis (two males, three females). Four patients had alcohol related cirrhosis and one had PBC. They had greater impairment of liver function but did not show signs of overt hepatic encephalopathy or ascites at the time of the MRS study. Mean plasma urea level was 4.5 (range 4.1-6.0) $\mathrm{mmol} / 1$. None of the patients in this group had any recent oesophageal variceal haemorrhage. Fasting plasma glucose levels were normal in all subjects and none had biochemical evidence of renal insufficiency.

STUDY PROTOCOL

Before each subject was positioned in the centre of the MR machine, intravenous access was gained via the antecubital veins of both arms using an 18 gauge cannula. The left arm was used for infusion of L-alanine and the right for sampling of blood. Blood was drawn at 15-20 minute intervals. Each subject had continuous electrocardiograph monitoring, and blood pressure and pulse were recorded at five minute intervals. After obtaining a sample of blood for initial biochemical measurements and obtaining three baseline ${ }^{31} \mathrm{P}$ MR spectra, a solution of pyrogen free L-alanine $(1.12 \mathrm{~mol} / \mathrm{l})$ (Boots Co., Nottingham, UK), prewarmed to $35^{\circ} \mathrm{C}$ in a water bath, was infused at a dose of $2.8 \mathrm{mmol} / \mathrm{kg}$ over a five minute period. ${ }^{31} \mathrm{P} \mathrm{MR}$ spectra were then acquired continuously for the next 90 minutes.

IN VIVO HEPATIC ${ }^{31} \mathrm{P}$ MRS

Hepatic ${ }^{31} \mathrm{P} M R$ spectra were acquired using a 1.5 T Eclipse MR machine (Marconi Medical Systems Inc, Cleveland, Ohio, USA). An enveloping transmitter body coil and a separate $15 \mathrm{~cm}$ surface coil, which was placed over the surface of the liver in the anterior axillary line, were used, both of which were double tuned for protons at $64 \mathrm{MHz}$ and for phosphorus at 
$26 \mathrm{MHz}$. The proton signal was used for shimming and to obtain a $T_{1}$ weighted image in the axial plane to confirm spatial localisation. Localised spectra were obtained from eight parasagittal planes each of nominal width of 30 $\mathrm{mm}$, using a two dimensional chemical shift imaging (2D-CSI) technique. ${ }^{15} 16$ Data were collected with a repetition time of $1000 \mathrm{~ms}$ (16 averages) and a pulse angle of $45^{\circ}$. Sequential spectra were obtained continuously at five minute intervals for 90 minutes following infusion of L-alanine.

\section{DATA PROCESSING}

A single observer (MAK) who was blinded to the patient's clinical condition analysed all data. The spectra were processed with a cosine filter in the spatial domain and a $60 \mathrm{~ms}$ exponential ( $5 \mathrm{~Hz}$ Lorentzian) filter in the time domain. After Fourier transformation, all data were manually phased. A sophisticated lineshape fitting analysis program (FITPLA) was employed which used a complex Lorentzian as a model function for each resonance. ${ }^{17}{ }^{18}$ The baseline roll caused by the delay in applying the localising phase encoding gradients in the 2D-CSI sequence made it necessary to perform the lineshape fitting analysis in three different phases. All spectra were baseline corrected using a baseline artefact subtraction
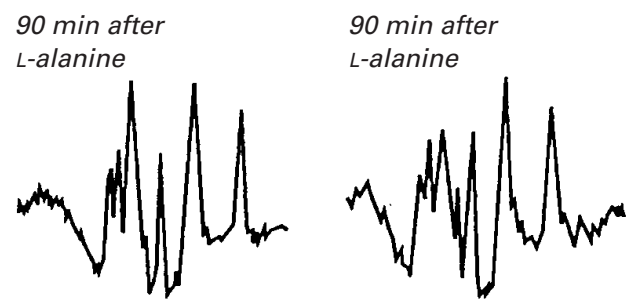

90 min after L-alanine

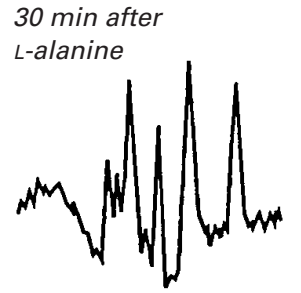

30 min after L-alanine
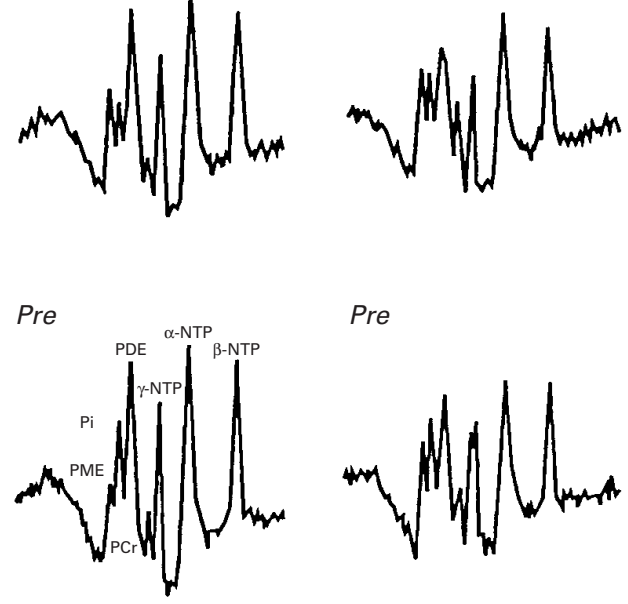

Pre

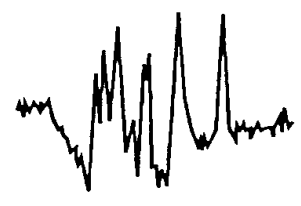

Compensated
30 min after L-alanine
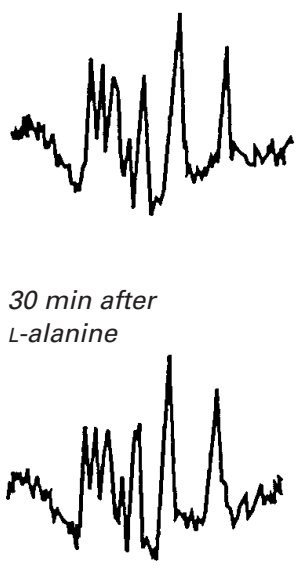

Pre

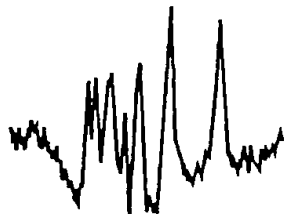

Decompensated

Figure 1 Typical phosphorus-31 magnetic resonance spectra obtained from a healthy volunteer, a patient with well compensated cirrhosis, and a patient with decompensated cirrhosis prior (Pre) to and after administration of L-alanine. Significantly elevated phosphomonoesters (PME) and reduced phosphodiesters (PDE) in both patient groups compared with controls at baseline is evident. Following administration of L-alanine, there was a significant increase in PME resonance in both healthy volunteers and patients with compensated cirrhosis which returned to baseline values after 90 minutes. Patients with decompensated cirrhosis showed increased PME at baseline which remained virtually unchanged after L-alanine. $P_{i}$, inorganic phosphate; NTP, nucleotide triphosphate.

(BLAST) technique. ${ }^{19}$ Baseline spectra were added together to improve the signal to noise ratio and analysed using the following lineshapes:

(I) PME $+\mathrm{P}_{\mathrm{i}}+\mathrm{PDE}+\mathrm{PCr}$ : a Lorentzian lineshape was assigned for each metabolite without the intensity, half linewidth, and chemical shift preset. A model lineshape was then constructed for this complex of metabolites.

(ii) $\alpha-\mathrm{NTP}$ and $\gamma$-NTP: two Lorentzian lineshapes were assigned with the respective half linewidths, intensities, and chemical shifts fixed as these parameters remained constant. (iii) $\beta$-NTP: three Lorentzian lineshapes were assigned with relative half linewidths, intensities, and chemical shifts fixed.

The same model lineshape was used for each NTP resonance in all spectra. ${ }^{18}$ Owing to the fact that NTP values may change following alanine infusion, all MRS data are expressed as a percentage of the total ${ }^{31} \mathrm{P}$ MRS signal in this dynamic study ${ }^{20}$ rather than as a ratio to $\beta$-NTP, which is an alternative standard methodology more suited to single non-dynamic measurements. ${ }^{21}$ The use of external reference standards for absolute quantification purposes was precluded, owing to the requirement for additional scanning time in what was already a lengthy MR study for these patients.

\section{BIOCHEMICAL MEASUREMENTS}

Plasma was assayed for alanine, insulin, glucagon, lactate, and urea using standard immunohistochemistry, high pressure liquid chromatography, and biochemical techniques. Capillary ammonia was measured using a Blood Ammonia Checker II (Kyoto Daiichi Kagaku Co., Ltd, Kyoto, Japan). Glucose levels were measured using an automated glucose analyser (RA-1000; Technicon Instrument Co. Ltd., Basingstoke, UK).

\section{STATISTICS}

The statistical significance of biochemical or MRS changes within a patient or volunteer group was calculated using one way analysis of variance. Differences in baseline values of individual variables were calculated using the Mann-Whitney test. Changes in individual metabolites following infusion of alanine were calculated using an "area under the curve" (AUC) method. The statistical significance of the differences in AUC between groups was calculated for each variable using the MannWhitney U test; p values less than 0.05 were considered to be statistically significant.

\section{Results \\ ${ }^{31} \mathrm{P}$ MRS \\ Baseline values}

Typical baseline in vivo ${ }^{31} \mathrm{P}$ MR spectra obtained prior to L-alanine infusion are illustrated in fig 1 . The most prominent differences between patients and healthy volunteers were in the PME and PDE resonances. There was a marked elevation in \% PME by $35 \%(p<0.01)$ in compensated cirrhosis and by $57 \%(\mathrm{p}<0.01)$ in decompensated cirrhosis compared with normal resting values (fig 2A). Baseline \% PDE values were significantly decreased in patients 

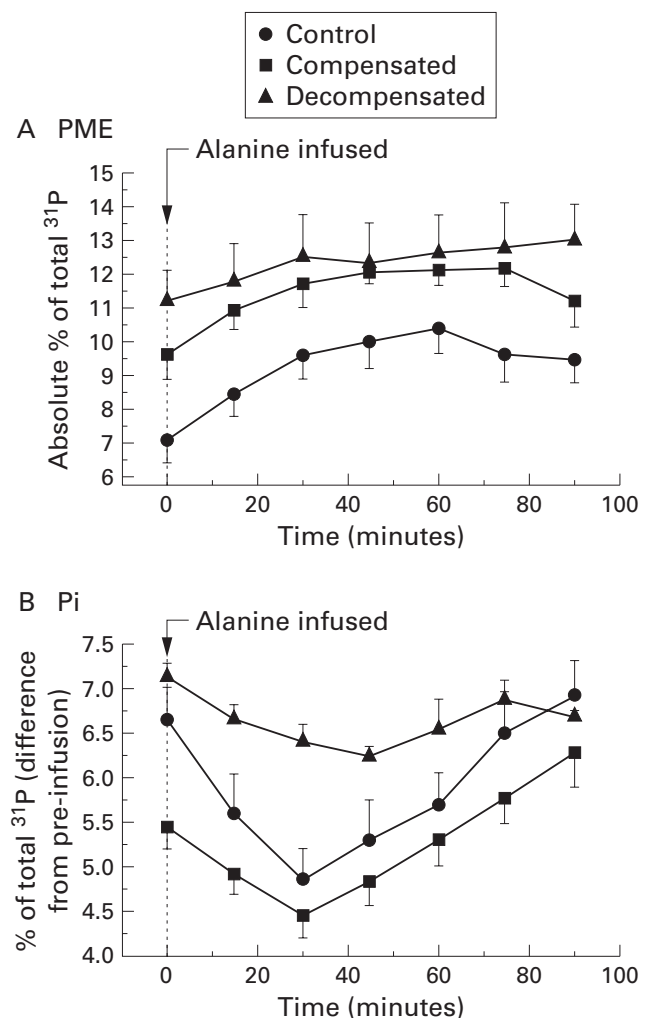

Figure 2 Changes in (A) percentage phosphomonoester $(\% P M E)$ and $(B)$ percentage inorganic phosphate $(\% P$ ) after infusion of L-alanine in healthy volunteers, and in patients with compensated cirrhosis and decompensated cirrhosis.

with decompensated cirrhosis $(\mathrm{p}<0.05 v$ healthy volunteers and $\mathrm{p}<0.05 v$ compensated patients). There was a non-significant reduction in baseline \%PDE values in the compensated cirrhosis group compared with healthy volunteers. Compared with healthy volunteers, baseline $\% \mathrm{P}_{\mathrm{i}}$ values were significantly lower in compensated patients $(p<0.008)$ and significantly higher in decompensated patients $(\mathrm{p}<0.01)$ (fig $2 \mathrm{~B}) . \% \mathrm{NTP}$ values were similar in all three groups.

PME CHANGES FOLLOWING L-ALANINE INFUSION (FIG 2A)

An increase in \%PME with a reciprocal decrease in $\% \mathrm{P}_{\mathrm{i}}$ was observed in healthy volunteers. \% PME levels increased from a preinfusion value of $7.16(0.70)$ to a maximum of 10.47 (0.76), an increase of $46 \%(p<0.01)$. Following L-alanine infusion, \%PME values were virtually unchanged in the decompensated cirrhosis group with baseline \%PME values of $11.22(0.90)$ and postinfusion values of 13.08 (1.06) $(p=0.47)$. In patients with compensated cirrhosis, \% PME increased by $27 \%(\mathrm{p}<0.03)$ from $9.65(0.75)$ to a maximum of 12.25 (0.55). The AUC for \%PME from baseline values was significantly greater in healthy volunteers compared with patients with decompensated cirrhosis $(\mathrm{p}<0.008)$. The AUC for $\% \mathrm{PME}$ was also significantly greater in compensated cirrhosis compared with patients with decompensated cirrhosis $(\mathrm{p}<0.03)$.
$P_{\mathrm{i}}$ CHANGES FOLLOWING L-ALANINE INFUSION (FIG 2B)

In healthy volunteers, $\% \mathrm{P}_{\mathrm{i}}$ decreased by $27 \%$ from a preinfusion value of $6.65(0.36)$ to a minimum of $4.86(0.34)(p<0.003)$ following L-alanine infusion. In patients with compensated cirrhosis, $\% \mathrm{P}_{\mathrm{i}}$ decreased from $5.44(0.26)$ at baseline to a minimum of $4.46(025)$, a fall of $18 \%(\mathrm{p}<0.02)$. Patients with decompensated cirrhosis showed a decline of $12 \%(\mathrm{p}<0.05)$ from $7.12(0.16)$ to $6.24(0.11)$. The AUC for $\% \mathrm{P}_{\mathrm{i}}$ from baseline values was significantly different in healthy volunteers compared with patients with decompensated cirrhosis $(p<0.008)$ but the AUC for $\% P_{i}$ was not significantly different between healthy volunteers and patients with compensated cirrhosis. The AUC for $\% \mathrm{P}_{\mathrm{i}}$ was also significantly different for patients with compensated cirrhosis compared with patients with decompensated cirrhosis $(p<0.008)$.

\%PDE AND \%NTP CHANGES FOLLOWING

L-ALANINE INFUSION

There was no net change in $\% \mathrm{PDE}$ or $\% \mathrm{NTP}$ in any patient or volunteer throughout the study. The AUC for $\%$ PDE or $\%$ NTP was not significantly different from baseline values between the three groups.

CHANGES IN PLASMA INSULIN (FIG 3)

Baseline plasma insulin levels were similar in healthy volunteers and decompensated cirrhosis patients. Following L-alanine infusion both groups showed an increase in serum insulin levels, returning to preinfusion values $50-60$ minutes after alanine administration. The compensated cirrhosis group had a large variability in basal serum insulin levels, indicated by the large standard error bars in fig 3, but these values were significantly elevated compared with the two other groups $(\mathrm{p}<0.02)$. Insulin levels in the compensated group remained elevated throughout the study.

CHANGES IN PLASMA GLUCOSE (FIG 3)

At baseline, plasma glucose was not significantly different in the three groups. Following L-alanine infusion, both healthy volunteers and patients with compensated cirrhosis showed a net increase in circulating glucose which reached a peak at 35 minutes and then returned to preinfusion values. Glucose levels fell in patients with decompensated cirrhosis, returning to preinfusion levels at 90 minutes. The AUC for plasma glucose was not significantly different from baseline values in the three study groups.

CHANGES IN PLASMA GLUCAGON (FIG 3)

Basal serum glucagon levels were elevated in both patient groups compared with healthy volunteers but only the compensated group showed a significant difference $(p<0.05)$. After L-alanine infusion, healthy volunteers showed a steady but significant $(\mathrm{p}<0.01)$ increase in serum glucagon levels from 9.1 (1.0) to 17.9 (1.7) pmol/1. The compensated group showed a significant increase from $12.0(0.8)$ to 15.6 (0.5) $\mathrm{pmol} / \mathrm{l}(\mathrm{p}<0.01)$ immediately following 

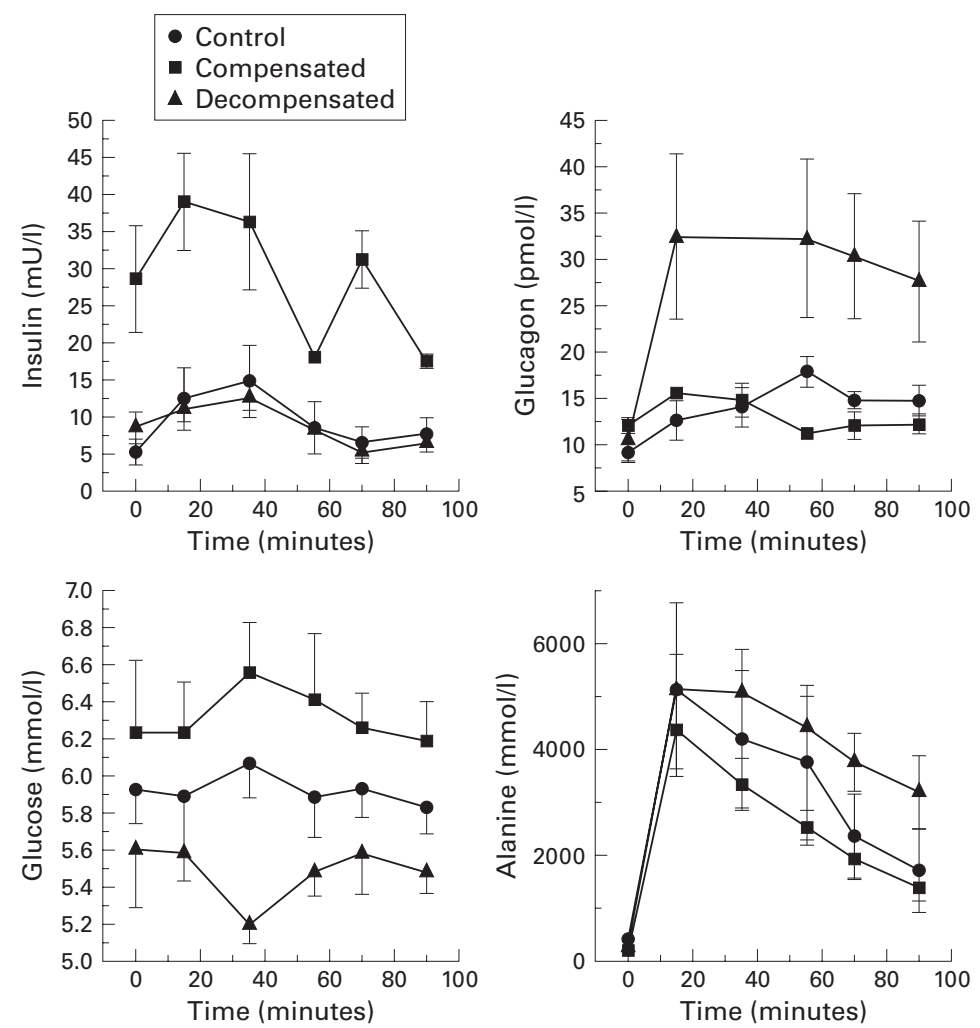

Figure 3 Plasma insulin, glucagon, glucose, and alanine concentrations in healthy volunteers, and in patients with compensated and decompensated cirrhosis prior to and after administration of L-alanine.
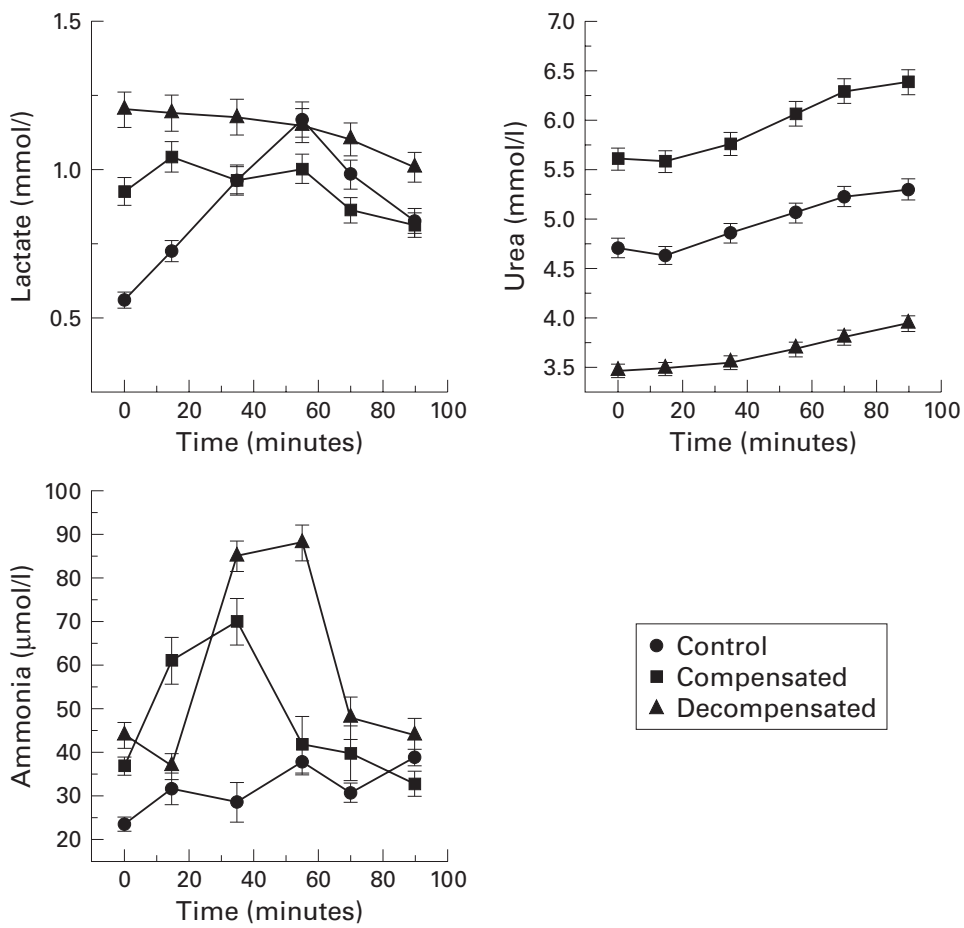

Figure 4 Plasma lactate, plasma urea, and capillary ammonia concentrations in healthy volunteers and in patients with compensated and decompensated cirrhosis prior to and after administration of L-alanine.

infusion but thereafter levels were similar to basal values. Decompensated patients had variable levels of serum glucagon, as indicated by the large error bars in fig 3. Following alanine infusion, glucagon levels remained elevated at approximately $30 \mathrm{pmol} / 1$ which was significantly higher $(\mathrm{p}<0.05)$ than in healthy volunteers and compensated patients.

CHANGES IN PLASMA ALANINE (FIG 3)

Baseline plasma alanine concentrations were not significantly different between the three study groups. Plasma alanine levels following infusion were very varied within each group, producing large standard errors from the mean. Both the compensated cirrhosis group and healthy volunteers had similar plasma alanine disappearance profiles. The decompensated cirrhosis group had an elevated plasma alanine level throughout the study. The AUC for plasma alanine was not significantly different from baseline values in the three study groups.

CHANGES IN PLASMA UREA (FIG 4)

At baseline, plasma urea was significantly lower in patients with decompensated liver disease compared with both healthy volunteers and patients with compensated liver disease $(p<0.05$ and $p<0.05$, respectively). No significant differences in basal urea concentrations were detected between healthy volunteers and patients with compensated cirrhosis. Following infusion of L-alanine, plasma urea concentration increased in all subjects but there were no statistically significant differences between the three groups. The AUC in plasma urea was significantly less in patients with decompensated cirrhosis compared with healthy volunteers and patients with compensated cirrhosis $(\mathrm{p}<0.05$ and $\mathrm{p}<0.05$, respectively). No significant differences were observed between healthy volunteers and compensated cirrhosis patients.

CHANGES IN CAPILLARY AMMONIA (FIG 4)

Baseline capillary ammonia levels were significantly higher $(\mathrm{p}<0.001)$ in compensated $(37$ (2) $\mu \mathrm{mol} / \mathrm{l})$ and decompensated (44 (3) $\mu \mathrm{mol} / \mathrm{l})$ cirrhosis patients than in healthy controls (24 (1.6) $\mu \mathrm{mol} / \mathrm{l})$. Following L-alanine administration, both patient groups showed an almost doubling of capillary ammonia levels $(p<0.05$ for compensated cirrhosis and $\mathrm{p}<0.03$ for decompensated cirrhosis). No significant changes were observed in healthy volunteers $(p=0.2)$. The AUC for capillary ammonia was significantly different in patients with decompensated cirrhosis compared with healthy volunteers and patients with compensated cirrhosis ( $\mathrm{p}<0.009$ and $\mathrm{p}<0.009$, respectively). A significantly greater change was also observed in compensated cirrhosis patients compared with healthy volunteers $(\mathrm{p}<0.05)$.

CHANGES IN PLASMA LACTATE (FIG 4)

Baseline levels of plasma lactate were significantly lower in healthy volunteers compared with patients in the compensated $(\mathrm{p}<0.01)$ and decompensated cirrhosis $(\mathrm{p}<0.001)$ groups. Following infusion of $\mathrm{L}$-alanine, plasma lactate levels increased significantly in healthy volunteers $(p<0.05)$, a level which was equivalent to baseline values in patients with decompensated cirrhosis. A steady decline towards preinfusion values then followed. The two patient groups showed very little change following infusion of 
L-alanine. The AUC for plasma lactate was significantly different in healthy volunteers compared with patients with decompensated cirrhosis $(\mathrm{p}<0.05)$. No significant differences were observed between the other groups.

\section{Discussion}

This is the first study to apply in vivo hepatic ${ }^{31} \mathrm{P}$ MRS as a non-invasive tool to determine dynamic changes in PME resonance in patients with cirrhosis of varying severity following bolus infusion of the progluconeogenic amino acid L-alanine. The combination of hepatic ${ }^{31} \mathrm{P}$ MRS and measurement of plasma alanine and glucose indicate changes in hepatic gluconeogenesis. ${ }^{12} 13$ Our results also suggest that changes associated with the PME and $P_{i}$ regions of the ${ }^{31} \mathrm{P}$ MR spectra following infusion of L-alanine may help differentiate between patients with compensated and decompensated cirrhosis and healthy subjects.

PME resonance is multicomponent and its constituents cannot be completely resolved using clinical MR systems, but at higher magnetic field strengths in vitro MRS techniques provide the required spectral definition to separate these metabolites. In vitro MRS methods on human tissue extracts have been used to study the metabolite changes responsible for in vivo PME signals in normal liver. ${ }^{22}$ This technique has also been applied by our group to the study of patients with cirrhosis. ${ }^{10}$ PME resonance includes contributions from the precursors of phospholipid membrane synthesis PC and $\mathrm{PE}^{1022-24}$ as well as contributions from AMP and phosphorylated intermediates of the gluconeogenic and glycolytic pathways. $^{102224}$

Our previous studies established the feasibility of using in vivo hepatic ${ }^{31} \mathrm{P}$ MRS as a non-invasive indication of gluconeogenesis in healthy human volunteers and animals through real time measurement of changes in PME resonance which reflects hepatic production of gluconeogenic intermediates such as 3PG, phospho-enol pyruvate, and G6P following L-alanine challenge. ${ }^{912}$ The technique has been validated in cachectic cancer patients using simultaneous ${ }^{31} \mathrm{P}$ MRS of the liver and glucose turnover measurements with labelled glucose and alanine. ${ }^{13}$ The elevation in PME resonance following intravenous L-alanine infusion has been demonstrated to be related to increased gluconeogenesis rather than glycolysis. ${ }^{1325} 26$

As with previously published studies, ${ }^{912132526}$ the most obvious change in the current study was a rise in PME resonance with a reciprocal decrease in $P_{i}$ resonance. Baseline PME values in patients with decompensated cirrhosis were the most elevated and our previous cross sectional in vivo and in vitro ${ }^{31} \mathrm{P}$ MRS studies of the cirrhotic liver have shown that this elevated baseline PME resonance correlated with worsening functional severity of liver disease. ${ }^{24}$ These changes are related to increased generation of cell membrane precursors such as PC and PE in the failing liver. ${ }^{16}{ }^{24}$ However, following L-alanine infusion there was no significant change in this resonance in these patients but both healthy volunteers and patients with compensated cirrhosis showed a steady PME increase which reached a maximum after 60 and 40 minutes, respectively. This dynamic MRS change was probably caused by increased production of gluconeogenic intermediates such as $3 \mathrm{PG}$ and G6P in the liver. These observations have been demonstrated previously in the rat $^{12}$ and in patients with lung cancer. ${ }^{132526}$ Unlike our study where it was not possible to use isotope tracer studies to confirm the MRS findings, the series of studies on patients with cancer cachexia have confirmed using tracer methodologies that the PME changes represent altered hepatic gluconeogenesis following L-alanine infusion. ${ }^{13}{ }^{26}$ The negligible PME response in patients with decompensated cirrhosis suggests that the gluconeogenic capacity of the cirrhotic liver was already at maximal capacity.

Increased hepatic metabolic activity, including $\beta$-oxidation of fatty acids and gluconeogenesis, is associated with a reduction in the MR invisible mitochondrial $P_{i}$ pool and also the cytoplasmic $\mathrm{P}_{\mathrm{i}}$ pool which can be detected non-invasively by ${ }^{31} \mathrm{P}$ MRS. ${ }^{27}$ Unlike the multicomponent PME resonance, $\mathrm{P}_{\mathrm{i}}$ resonance is not complicated by other metabolites and its fluctuations following L-alanine infusion are probably a better indicator of the true dynamics of hepatic metabolism following a gluconeogenic stimulus. In this study, patients with compensated cirrhosis exhibited significantly reduced baseline $\% \mathrm{P}_{\mathrm{i}}$ values compared with values in healthy volunteers. This observed reduction may be attributable to increased basal gluconeogenic rates in patients with compensated cirrhosis which could also be inferred from the elevated baseline plasma glucose levels and \%PME values compared with healthy volunteers. However, following a metabolic challenge with L-alanine infusion, there was a significant decrease from baseline in $\% \mathrm{P}_{\mathrm{i}}$ values in all subjects which was least remarkable in patients with decompensated cirrhosis. This is consistent with the lack of any significant change in \% PME in this patient group.

The mechanism of increased gluconeogenesis in cirrhosis remains unclear. This may be the result of reduced glycogen stores, a defect of glycogenolysis, or an alteration in the insulin/glucagon ratio. Petersen and colleagues ${ }^{6}$ have shown a $34 \%$ reduction in hepatic glycogen stores and a reduction of 3.5-fold in the rate of glycogenolysis in patients with well compensated cirrhosis compared with healthy volunteers. It would be reasonable to hypothesise that patients with decompensated liver disease may have a more substantial reduction in glycogen stores and a more profound reduction in the rates of glycogenolysis. In order to be able to provide adequate amounts of glucose for peripheral metabolism, gluconeogenesis must be increased in the context of reduced generation of glucose from glycogen. Kabadi has suggested that reduced hepatic glycogen stores may be the regulator of increased glucagon concentration observed in patients with cirrhosis. $^{5}$ 
The patient group that we studied with compensated cirrhosis had hyperinsulinaemia which is thought to be related to decreased hepatic metabolism of insulin ${ }^{28}$ whereas patients with decompensated cirrhosis had much lower insulin levels, probably because they were unable to mount a gluconeogenic response with exogenously administered L-alanine. These observed changes in insulin/ glucagon ratio in addition to insulin resistance may contribute to the increase both in basal gluconeogenesis and that observed after bolus infusion of L-alanine in patients with compensated cirrhosis. ${ }^{29}$

The most striking observation in this study was in patients with decompensated cirrhosis. This patient group exhibited no increase in $\%$ PME following infusion of L-alanine, which probably reflects maximal gluconeogenesis, despite elevated levels of plasma glucagon compared with the other two groups. The mechanism of the increase in glucagon concentration following L-alanine infusion is likely to be due to a direct stimulatory effect of L-alanine on glucagon secretion. ${ }^{30}$ The lack of any gluconeogenic response to hyperglucagonaemia suggests resistance to the action of glucagon in decompensated cirrhosis. The underlying pathophysiological basis of this resistance has been postulated to be due to lack of glycogen stores. This supposition has not been supported by the results of a recent study of whole body gluconeogenesis in patients with cirrhosis using stable isotope technology, which confirmed that these patients have increased gluconeogenesis. ${ }^{8}$ However, the glycaemic response to glucagon infusion was blunted despite repletion of glycogen stores. This observation suggests that the mechanism underlying the observed glucagon resistance is unlikely a result of reduced glycogen stores but may be due to a receptor or postreceptor defect. The latter is more probable because patients with cirrhosis have resistance to multiple hormone systems including epinephrine and insulin. ${ }^{29}{ }^{31}$ Tabaru and colleagues ${ }^{32}$ investigated the glycaemic response to bolus infusion of branched chain amino acids in patients with cirrhosis of varying severity and observed an exaggerated glycaemic response in patients with compensated disease and a minimal glycaemic response in patients with decompensated disease. This is consistent with the results of our study. The increment in plasma insulin concentration was similar to that observed in our study suggesting insulin resistance in patients with compensated cirrhosis. In contrast, patients with decompensated disease showed increments in plasma glucagon concentration which were similar to those observed in our study which are suggestive of glucagon resistance. Taken together, it is likely that the predominant derangement in carbohydrate metabolism in patients with compensated cirrhosis is insulin resistance whereas glucagon resistance prevails as the predominant abnormality in patients with decompensated liver disease.

Numerous previous studies have shown a progressive reduction in urea synthesis in patients with increasing severity of liver disease which is refractory to glucagon infusion and is likely to reflect reduced hepatic function in patients with cirrhosis. ${ }^{8334}$ Changes in the concentration of ammonia were appropriate and expected in healthy volunteers and patients with compensated cirrhosis. The increase in ammonia following L-alanine infusion is likely to result from transamination of alanine which is then channelled into the gluconeogenic and other pathways. ${ }^{9}{ }^{12}$

${ }^{31} \mathrm{P}$ MRS highlights the differences in metabolic response between patients with compensated and decompensated cirrhosis following bolus infusions of L-alanine. The elevation in $\% \mathrm{PME}$ and the reciprocal reduction in $\% \mathrm{P}_{\mathrm{i}}$ provides good differentiation between healthy volunteers and patients with compensated and decompensated cirrhosis. These results indicate increased basal gluconeogenesis in compensated cirrhosis and impaired capacity of the failing liver to increase gluconeogenesis following infusion of the progluconeogenic substrate L-alanine, in decompensated cirrhosis. This observation may explain the tendency of patients with decompensated disease to develop hypoglycaemia when placed in a stressful environment.

In vivo hepatic ${ }^{31} \mathrm{P}$ MRS provides a noninvasive indication of hepatic gluconeogenesis following L-alanine infusion. The technique has now been shown to be valuable in studies of cancer cachexia and cirrhosis and may have wider applications in assessing other disease states where gluconeogenic control is impaired, such as diabetes mellitus. This study was supported by the Medical Research Council
(UK) and Marconi Medical Systems Inc (Cleveland, Ohio, USA). We would like to thank Dr Jason A Wilson and Ms Jane $\mathrm{E}$ Schwieso for help in performing this study. Ms Jennifer Porina, Mrs Mary Crisp, Mrs Dulcie Rodrigues, and Mrs Porina, Mrs Mary Crisp, Mrs Dulcie Rodrigues, and Mrs Joanna Allsop helped prepare the illustrative material. We are
also grateful to Professor Desmond Johnston and Dr Janice Main from Imperial College School of Medicine for useful disMain from
cussions.

1 McCullough AJ, Tavill AS. Disordered energy and protein McCullough AJ, Tavill AS. Disordered energy and protein 77 .

2 Krahenbuhl S, Weber FL Jr, Brass EP. Decreased hepatic glycogen content and accelerated response to starvation in rats with carbon tetrachloride-induced cirrhosis. Hepatology 1991;14:1189-95.

3 Schneeweiss B, Graninger W, Ferenci P, et al. Energy metabolism in patients with acute and chronic liver disease. Hepatology 1990;11:387-93.

4 Müller MJ, Läutz HU, Plogmann B, et al. Energy expenditure and substrate oxidation in patients with cirrhosis: the impact of cause, clinical staging and nutritional state. Hepatology 1992;15:782-94.

5 Kabadi UM. Is hepatic glycogen content a regulator of glucose secretion? Metabolism 1992;41:113-15.

6 Petersen K, Krssak M, Navarro V, et al. Contributions of net Petersen $\mathrm{K}, \mathrm{Krssak} M$, Navarro V, et al. Contributions of net
hepatic glycogenolysis and gluconeogenesis to glucose prohepatic glycogenolysis and gluconeogenesis

7 Cavallo-Perin P, Cassader M, Bozzo C, et al. Mechanism of insulin resistance in human liver cirrhosis. Evidence of a combined receptor and postreceptor defect. F Clin Invest 1985;75:1659-65.

8 Bugianesi E, Kalhan S, Burkett E, et al. Quantification of gluconeogenesis in cirrhosis: response to glucagon. Gastroenterology 1998;115:1530-40.

9 Dagnelie PC, Menon DK, Cox IJ, et al. Effect of L-alanine infusion on ${ }^{31} \mathrm{P}$ nuclear magnetic resonance spectra of normal human liver: towards biochemical pathology in vivo. Clin Sci (Colch) 1992;83:183-90.

10 Taylor-Robinson SD, Thomas EL, Sargentoni J, et al. Cirrhosis of the human liver: An in vitro ${ }^{31} \mathrm{P}$ nuclear magnetic resonance study. Biochim Biophys Acta 1995; magnetic reson

11 Ross BD, Hems R, Krebs HA. Carbohydrate metabolism of the perfused rat liver. Biochem $\mathcal{F} 1967 ; 102: 942-51$. 
12 Changani $\mathrm{K}$, Barnard M, Bell J, et al. In vivo assessment of metabolic perturbations after alanine and glucagon admin-
istration using ${ }^{31} \mathrm{P}$ MRS in the rat. Biochim Biophys Acta istration using ${ }^{31} \mathrm{P} M$

13 Leij-Halfwerk S, van den Berg JW, Sijens PE, et al. Altered hepatic gluconeogenesis during L-alanine infusion in weight-losing lung cancer patients as observed by phosphorus magnetic resonance spectroscopy and turnover measurements. Cancer Res 2000;60:618-23.

14 Pugh RNH, Murray-Lyon IM, Dawson JL, et al. Transection of the oesophagus for bleeding oesophageal varices. $B$ 7 Surg 1973;60:646-9.

15 Cox IJ, Menon DK, Sargentoni J, et al. Phosphorus-31 magnetic resonance spectroscopy of the human liver using chemical shift imaging techniques. F Hepatol 1992:14:26575.

16 Menon DK, Sargentoni J, Taylor-Robinson SD, et al. Effect of functional grade and aetiology on in vivo hepatic phosphorus-31 magnetic resonance spectroscopy in cirrhosis: biochemical basis of spectral appearances. Hepatology 1995;21:417-27.

17 Ala-Korpela M, Korhonen A, Keisala J, et al. ${ }^{1} \mathrm{H}$ NMR-based absolute quantitation of human lipoprotein and their lipid contents directly from plasma. $\mathcal{F}$ Lipid Res 1994;35:2292-304.

18 Changani K, Ala-Korpela M, Fuller BJ, et al. Importance of concise metabolite prior knowledge for data analysis: Biochemical implications of dynamic ${ }^{31} \mathrm{P}$ NMR ex vivo pis liver studies. NMR Biomed 1999;12:1-8.

19 Saeed N, Menon D. A knowledge-based approach to minimize baseline roll in chemical shift imaging. Magn Reson Med 1993;29:591-8.

20 Taylor-Robinson SD, Buckley C, Changani KK, et al. Cerebral proton and phosphorus-31 magnetic resonance spectroscopy in patients with subclinical hepatic encephalopathy. Liver 1999;19:389-98.

21 Taylor-Robinson SD, Sargentoni J, Bell JD, et al. In vivo and in vitro hepatic phosphorus-31 magnetic resonance spectroscopy and electron microscopy in chronic ductopenic troscopy and electron microscopy in chronic ductopenic

22 Bell JD, Cox IJ, Sargentoni J, et al. A ${ }^{31} \mathrm{P}$ and ${ }^{1} \mathrm{H}$ NMR investigation in vitro of normal and abnormal human liver. investigation in vitro of normal and abn
Biochim Biophys Acta 1993;1225:71-7.
23 Ruiz-Cabello J, Cohen JS. Phospholipid metabolites as indicators of cancer cell function. NMR Biomed 1992;5:22633 .

24 Taylor-Robinson SD, Sargentoni J, Bell JD, et al. In vivo and in vitro hepatic ${ }^{31} \mathrm{P}$ magnetic resonance spectroscopy and electron microscopy of the cirrhotic liver. Liver 1997;4: 198-209.

25 Leij-Halfwerk S, Dagnelie PC, Kappert P, et al. Decreased energy and phosphorylation status in the liver of lung cancer patients with weight loss. F Hepatol 2000;32:887-92.

26 Leij-Halfwerk S, Dagnelie PC, Van Den Berg JW, et al. Hepatic sugar phosphate levels reflect gluconeogenesis in lung cancer: simultaneous turnover measurements and ${ }^{31} \mathrm{P}$ magnetic resonance spectroscopy in vivo. Clin Sci (Colch) 2000;98:167-74.

27 Seery JP, Bryant DJ, Jenkinson G, et al. Intravenous intralipid infusion in hepatic steatosis: An in vivo hepatic phosphorus-31 magnetic resonance spectroscopy study. Hepatol Res 1998;12:50-8.

28 Yoshida T, Ninomiya K, Matsumoto T, et al. Glucagon and insulin metabolism in cirrhotic patients. Hepatogastroenterology 1998;45:468-71.

29 Merli M, Leonetti F, Riggio O, et al. Glucose intolerance and insulin resistance in cirrhosis are normalized after liver transplantation. Hepatology 1999;30:649-54.

30 Kuhara T, Ikeda S, Ohneda A, et al. Effects of intravenous infusion of 17 amino acids on the secretion of $\mathrm{GH}$, glucagon, and insulin in sheep. Am f Physiol 1991;260: E21-6.

31 Schricker T, Albuszies G, Weidenbach H, et al. Effects of epinephrine on glucose metabolism in patients with alcoholic cirrhosis. Hepatology 1996;24:330-6.

32 Tabaru A, Shirohara H, Moriyama A, et al. Effects of branched-chain-enriched amino acid solution on insulin and glucagon secretion and blood glucose level in liver cirrhosis. Scand 7 Gastroenterol 1998;33:853-9.

33 Fabri A, Marchesini G, Bianchi G, et al. Unresponsiveness of hepatic nitrogen metabolism to glucagon infusion in patients with cirrhosis: dependence on liver cell failure. patients with cirrhosis: depen
Hepatology 1993;18:28-35.

34 Bessman AN, Hawkins R. The relative effects of enterically administered plasma and packed cells on circulating blood ammonia. Gastroenterology 1963;45:368-73. 